\title{
Atmospheric thermal convection and strong chaotic fluctuations of global temperature on Earth and on Mars
}

\author{
A. Bershadskii \\ ICAR, P.O. Box 31155, Jerusalem 91000, Israel \\ bershads@gmail.com
}

\begin{abstract}
It is shown that the atmospheric thermal (buoyancy driven) convection plays the main role in generation of the strong chaotic fluctuations of the global temperature through the KolmogorovBolgiano-Obukhov mechanism (in the frames of the distributed chaos approach). It is valid for the planets with substantial atmosphere such as the Earth and Mars. Direct numerical simulations, the Berkeley Earth daily surface-atmospheric temperature dataset (over land) as well as the MACDA Mars reanalysis of the data inferred from the Thermal Emission Spectrometer measurements for the Mars Global Surveyor satellite were used in order to support this conclusion.
\end{abstract}

\section{INRODUCTION}

While the trends of the global (atmospheric) temperature are widely discussed its strong fluctuations attract much less interest. However, understanding of the nature of these fluctuations can be important for a meaningful forecast and analysis of the underlying physical processes. Recent developments in processing of the historical temperature data resulted in the good quality daily time series available for a statistical analysis [1], [2], that can be useful for understanding of the nature of these fluctuations.

The atmospheric thermal convection plays an important role in sustaining the global balance between the radiative heating by the Sun and cooling by the feedback thermal radiation to the outer space for the planets with substantial atmosphere (such as the Earth and Mars). While the trends of the global temperature are usually analysed in the pure deterministic (and linear) terms the situation with the strong fluctuations of the global atmospheric temperature is more complex. Apparently these fluctuations seem to be chaotic.

The deterministic chaos was discovered in a simple model of the thermal convection [3]. The area of applications covered by the deterministic chaos turned out to be vast (see, for instant, Ref. [4]). Naturally, the atmospheric thermal convection continues to be the main area of these applications. The question is: Could the strong fluctuations of the global temperature be described in the terms of the deterministic chaos? The pure deterministic chaos turned out to be restricted in its applicability to the real processes because the parameters of the real (natural) systems are themselves always randomly fluctuating. One can take into account this phenomenon using the notion of distributed chaos (see, for instance, Ref. [5]). For the buoyancy driven thermal convection the distributed chaos can be applied in the terms of the Kolmogorov-Bolgiano-Obukhov phenomenology [6]- [12].

The Section II provides a short introduction into the
Kolmogorov-Bolgiano-Obukhov phenomenology. In the Section III this phenomenology has been used in the frames of the distributed chaos approach. In the Section IV the theoretical results have been compared with the historical global temperature data (the Berkeley Earth daily data set of the surface-air temperature over land [1],[2]). In the Section V analogous comparison has been made with the Martian surface-atmospheric temperature fluctuations (the MACDA Mars reanalysis of the data inferred from the Thermal Emission Spectrometer measurements for the Mars Global Surveyor satellite).

\section{INERTIAL-BUOYANCY RANGE OF SCALES}

The thermal (buoyancy driven) convection in a plane horizontal layer in the Boussinesq approximation is described by the system of equations [13]

$$
\begin{gathered}
\frac{\partial \mathbf{u}}{\partial t}+(\mathbf{u} \cdot \nabla) \mathbf{u}=-\frac{\nabla p}{\rho_{0}}+\sigma g \theta \mathbf{e}_{z}+\nu \nabla^{2} \mathbf{u} \\
\frac{\partial \theta}{\partial t}+(\mathbf{u} \cdot \nabla) \theta=S \frac{\Delta}{H} e_{z} u_{z}+\kappa \nabla^{2} \theta \\
\nabla \cdot \mathbf{u}=\mathbf{0}
\end{gathered}
$$

where $\mathbf{u}$ is the velocity, $\theta$ is the temperature fluctuations, $p$ is the pressure, $\mathbf{e}_{z}$ is the a unit vector along the gravity, $g$ is the gravity acceleration, $H$ is the distance and $\Delta$ is the temperature difference between the layers, $\nu$ is the viscosity and $\kappa$ is the thermal diffusivity, $\rho_{0}$ is the mean density, $\sigma$ is the thermal expansion coefficient. For the unstable (Rayleigh-Bénard) stratification the coefficient $S=+1$ and for the stable stratification the coefficient $S=-1$.

In the non-viscous and non-diffusive case $(\nu=\kappa=0)$ this system has an invariant [13]

$$
\mathcal{E}=\int_{V}\left(\mathbf{u}^{2}-S \sigma g \frac{H}{\Delta} \theta^{2}\right) d \mathbf{r},
$$


where $V$ is the volume of the domain under consideration.

A generalization of the Kolmogorov-BolgianoObukhov approach [6]-[12] for the inertial-buoyancy range can relate the characteristic temperature fluctuations $\theta_{c}$ and the characteristic frequency $f_{c}$ using the dimensional considerations

$$
\theta_{c} \propto(\sigma g)^{-1} \varepsilon^{1 / 2} f_{c}^{1 / 2},
$$

where

$$
\varepsilon=\left|\frac{d\left\langle\mathbf{u}^{2}-S \sigma g \frac{d}{\Delta} \theta^{2}\right\rangle}{d t}\right|
$$

denotes the (generalized) dissipation/transfer rate and the $\langle\ldots\rangle$ denotes averaging over the spatial domain.

In the case of global thermal convection one should replace the plane horizontal layer by a thin spherical shell and take into account the global rotation term in the Eq. (1) (both these amendments conserve the generalised energy Eq. (4)). The global radiation and water in different phases (clouds etc.) for Earth and dust for Mars are much more difficult to account. However, all these additional factors can be integrated into a generalized dissipation/transfer rate $\varepsilon$ and the Kolmogorov-BolgianoObukhov like estimate Eq. (5) can be still preserved for an inertial-buoyancy range of scales (see below).

\section{TEMPORAL DISTRIBUTED CHAOS}

The exponential frequency spectrum

$$
E(f) \propto \exp \left(-f / f_{c}\right)
$$

is a typical spectrum for the deterministic (bounded and smooth) dynamical systems (see, for instance, Refs. [15], [16] and references therein).

A simplified model of the Rayleigh-Bénard convection (Lorenz system [3])

$$
\frac{d x}{d t}=\sigma(y-x), \quad \frac{d y}{d t}=r x-y-x z, \quad \frac{d z}{d t}=x y-b z,
$$

for instance, exhibits such spectrum for the parameters $b=8 / 3, r=28.0, \sigma=10.0$. Figure 1 shows the power spectrum of the $z$-component. The dashed straight line corresponds to the Eq. (7) in the semi-logarithmic scales.

For more complex situations the parameter $f_{c}$ can fluctuate and it is necessary to consider an ensemble average over this parameter to compute the power spectrum [5]

$$
E(f)=\int P\left(f_{c}\right) \exp -\left(f / f_{c}\right) d f_{c}
$$

In the frames of the Kolmogorov-Bolgiano-Obukhov phenomenology the distribution $P\left(f_{c}\right)$ can be readily

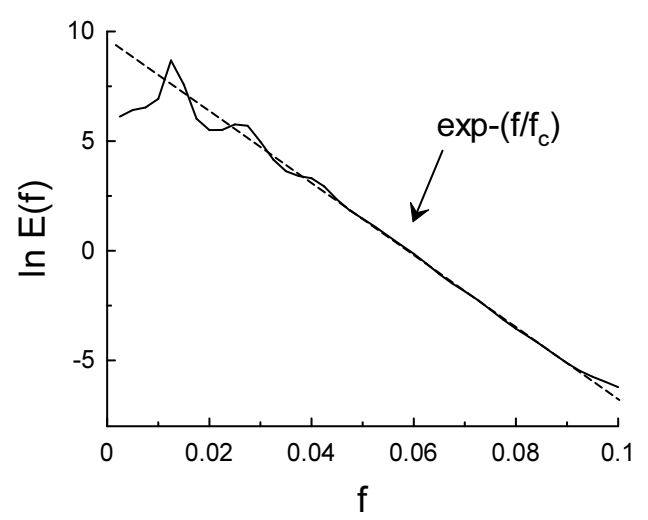

FIG. 1: Power spectrum of $z$-component for the Lorenz model.

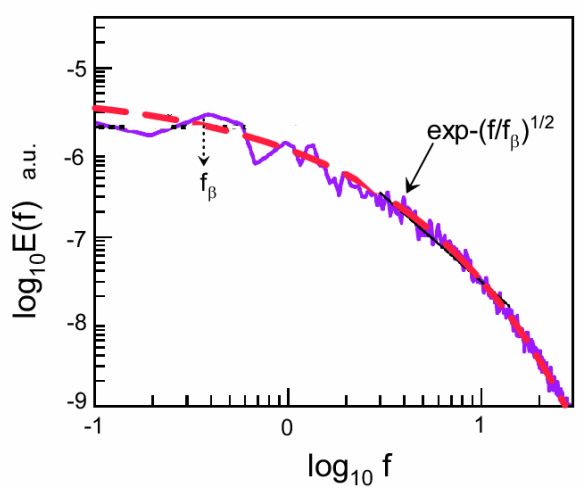

FIG. 2: Power spectrum of the temporal temperature fluctuations for the DNS of the the Rayleigh-Bénard convection.

found from the Eq. (5) in the case of Gaussian distribution of the characteristic temperature fluctuations $\theta_{c}$

$$
P\left(f_{c}\right) \propto f_{c}^{-1 / 2} \exp -\left(f_{c} / 4 f_{\beta}\right)
$$

where $f_{\beta}$ is a constant parameter.

Substituting the Eq. (9) into the Eq. (8) we obtain

$$
E(f) \propto \exp -\left(f / f_{\beta}\right)^{1 / 2}
$$

Figure 2 shows a temporal (frequency) power spectrum for the temperature fluctuations obtained in direct numerical simulations (DNS) of the system Eqs. (1-3) (the Rayleigh-Bénard convection conditions). The spectral data have been shown in the log-log scales and were taken from Fig. 7 of the the Ref. [17]. The measurements in these DNS were made using real-space probes located at the centre of a cubical box. The mean velocity at the centre of the cube has zero value. Therefore, the measurements with the real-space probes give real 


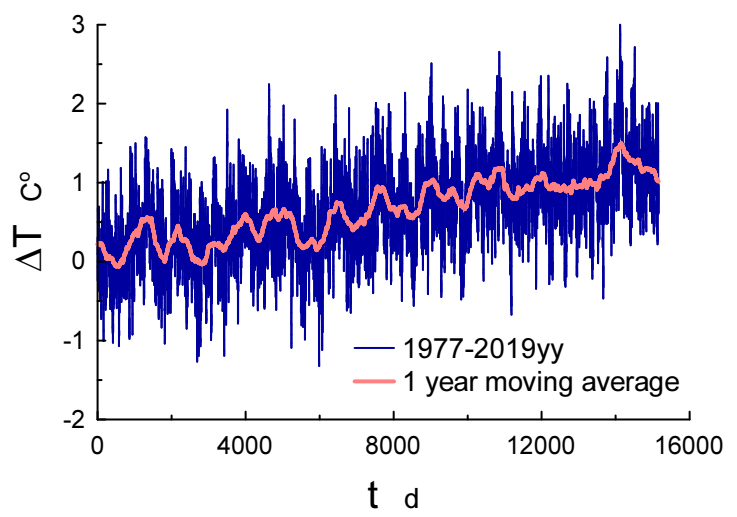

FIG. 3: Global average surface temperature anomaly for 19772019yy. The land-based daily data were taken from the Ref. [2].

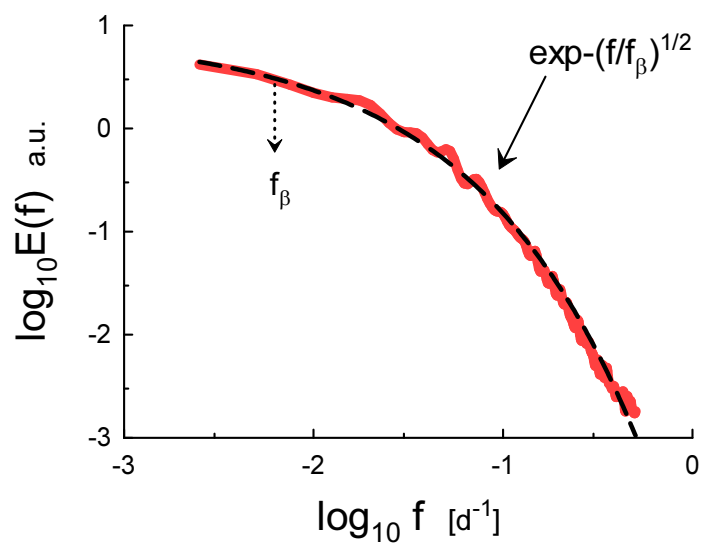

FIG. 4: Power spectrum corresponding to the detrended daily data for the period 1977-2019yy.

temporal (frequency) spectrum [17]. The Prandtl number was taken $P r=1$ (i.e. of the order usual for the air at the normal conditions) and the Rayleigh number was taken $R a=10^{8}$.

The dashed curve in the Fig. 2 indicates the stretched exponential spectrum Eq. (10) and the dotted vertical arrow indicates location of the frequency $f_{\beta}$. One can see that the distributed chaos is tuned to the coherent low-frequency oscillations with the frequency $f_{\beta}$.

\section{TEMPERATURE FLUCTUATIONS: EARTH}

The good quality daily historical time series of the global surface-air temperature over land are now avail-

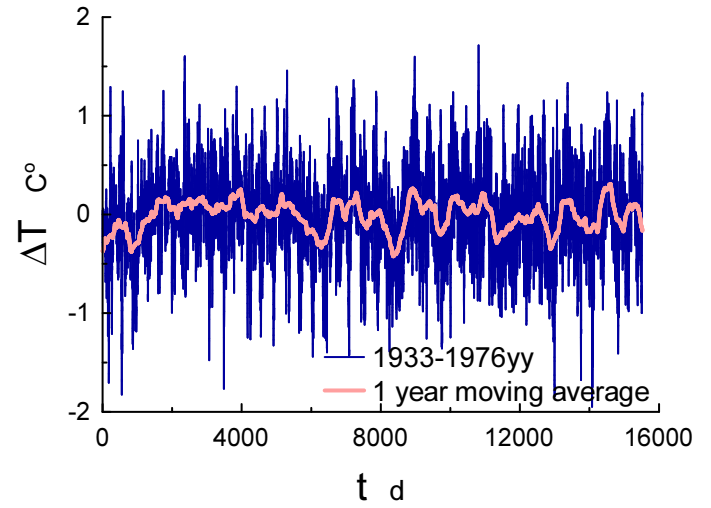

FIG. 5: Global average surface temperature anomaly for 19331976yy. The land-based daily data were taken from the Ref. [2].

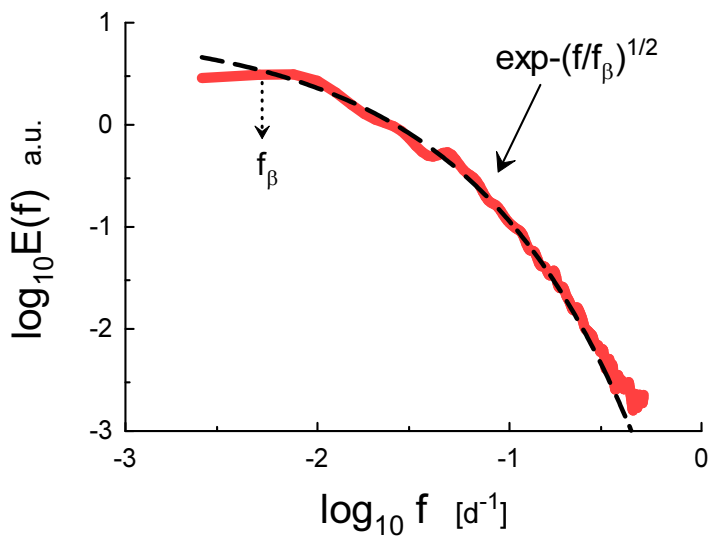

FIG. 6: Power spectrum corresponding to the detrended daily data for the period 1933-1976yy.

able for a statistical analysis [1],[2].

Figure 3 shows a global average surface-air temperature anomaly for the recent period 1977-2019yy (relative to the Jan. 1951 - Dec. 1980 average). The land-based daily data for this period were taken from the site [2]. Since the data set is statistically non-stationary a certain detrending is necessary before a spectral analysis. The detrending was produced by subtraction of the one year moving average of the data set (shown as the red line in the Fig. 3) from the original one. This subtraction removes the long-term trends and the remaining timeseries represent the daily to intra-annual dynamics only (cf. Refs. [18], [19] for such type of detrending).

Figure 4 shows power spectrum corresponding to the detrended data. The dashed line in the Fig. 4 indicates 


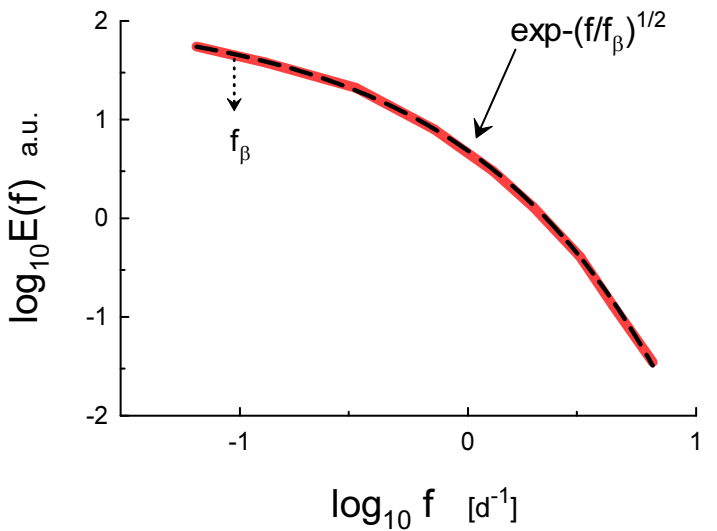

FIG. 7: Power spectrum of the global surface-atmospheric temperature fluctuations for Mars.

the stretched exponential decay Eq. (10). The dotted arrow indicates position of the scale $f_{\beta}$.

The data for a previous time period of the same length 1933-1976yy (taken from the same site [2]) were detrended by the same method - Fig. 5. Figure 6 shows corresponding power spectrum. The dashed line in the Fig. 6 indicates the stretched exponential decay Eq. (10). The dotted arrow indicates position of the scale $f_{\beta}$.

\section{TEMPERATURE FLUCTUATIONS: MARS}

Main physical properties of the 'climate and meteorology' related to the Martian atmosphere turned out to be surprisingly similar to those of the Earth atmosphere (see for a review Refs. [20, 21]) and references therein). Although the Martian atmosphere (consisting mostly of
$\mathrm{CO}_{2}$ ) is thinner than that of Earth the thermal (buoyancy driven) convection processes in this atmosphere are no less vigorous and substantial than those in the Earth atmosphere. The main difference in this respect is the strong influence of water (in different phases) on the Earth's atmospheric dynamics, whereas the dust is the rather significant factor in the Martian atmospheric dynamics. Therefore it is interesting to compare the above results obtained for Earth with those available now for Mars (cf. final remarks in the Section II).

Sun-synchronous 2-hour polar orbits (there was a $30^{\circ}$ displacing in longitude at every new orbit) of the NASA's Mars Global Surveyor with the Thermal Emission Spectrometer on board provided a well-sampled temporal and spatial coverage for the period from February 1999 to August 2004. The MACDA (Mars Analysis Correction Data Assimilation) reanalysis of the data inferred from the Thermal Emission Spectrometer measurements were then used to estimate the Martian surface-atmospheric temperature fluctuations [21], 22].

Figure 7 shows corresponding power spectrum (the spectral data were taken from Fig. $1 b$ of the Ref. [23]). The strong spectral spikes corresponding to the Mars diurnal cycle and its harmonics are not shown in the Fig. 7 (a day on Mars is approximately equal to that on Earth). The dashed line in the Fig. 7 indicates the stretched exponential decay Eq. (10). The dotted arrow indicates position of the scale $f_{\beta}$.

\section{ACKNOWLEDGEMENT}

I gratefully acknowledge use of the data provided by the Berkeley Earth dataset (USA).
[1] R. Rohde et al., Berkeley Earth Temperature Averaging Process. Geoinfor Geostat: An Overview 1:2. doi:10.4172/gigs.1000103 (2013).

[2] Available at: Complete_TAVG_daily.txt 20-Jan-2020, http://berkeleyearth.lbl.gov/auto/Global

[3] E.N. Lorenz, J. Atm. Sci., 20, 130 (1963)

[4] M. Cencini, F. Cecconi and A. Vulpiani, Chaos: From Simple Models to Complex Systems (World Scientific Publishing, 2010)

[5] A. Bershadskii, Res. Notes AAS, 4, 10 (2020)

[6] R. Bolgiano, J. Geophys. Res., 64, 2226 (1959)

[7] A.M. Obukhov, Dokl. Akad. Nauk SSSR, 125, 1246 (1959)

[8] A. S. Monin, A. M. Yaglom, Statistical Fluid Mechanics, Vol. II: Mechanics of Turbulence (Dover Pub. NY, 2007)

[9] I. Procaccia and R. Zeitak, Phys. Rev. Lett., 62, 2128 (1989)

[10] V.S. L'vov, Phys. Rev. Lett., 67, 687 (1991)

[11] G. Falkovich and V.S. L'vov, Physica D, 57, 85 (1992)
[12] E.S.C. Ching, Statistics and Scaling in Turbulent Rayleigh-Bénard Convection (Singapore: Springer, 2014)

[13] A. Kumar, A.G. Chatterjee and M.K. Verma, Phys. Rev. E, 90, 023016 (2014)

[14] M.K. Verma, A. Kumar and A. Pandey, New J. Phys., 19, $025012(2016)$

[15] U. Frisch and R. Morf, Phys. Rev., 23, 2673 (1981)

[16] N. Ohtomo, K. Tokiwano, Y. Tanaka et. al., J. Phys. Soc. Jpn. 641104 (1995)

[17] A. Kumar and M.K. Verma, R. Soc. open sci., 5, 172152 (2018)

[18] M.C. Wheeler and H.H. Hendon, Monthly Weather Review, 132, 1917 (2004).

[19] M.J. Ventrice et al., Monthly Weather Review, 141, 4197 (2013).

[20] P.L. Read, S.R. Lewis and D.P. Mulholland, Rep. Prog. Phys., 78, 125901 (2015)

[21] W. Chen, S. Lovejoy, and J.-P. Muller, J. Geophys. Res. Atmos., 121, 11968 (2016) 
[22] L. Montabone, K. Marsh, S.R. Lewis, P.L. Read, M.D. Smith, J. Holmes, A. Spiga, D. Lowe, and A. Pamment, Geosci. Data J., 1, 129 (2014)

[23] S. Lovejoy, L. Del Rio Amador, and R. Hebert, in
"Seasonal to Interannual Prediction System (StocSIPS)", A. Tsonis (eds) Advances in Nonlinear Geosciences (Springer, Cham 2017) 